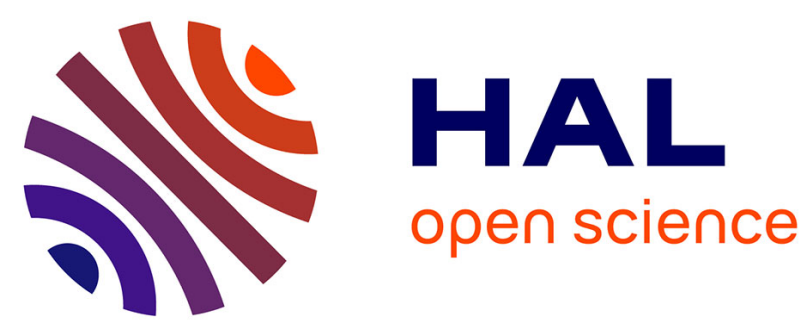

\title{
Flow patterns in the vicinity of triple line dynamics arising from a local surface tension model
}

\author{
Jerome Monnier, Adel Benselama, Iulian Cotoi
}

\section{To cite this version:}

Jerome Monnier, Adel Benselama, Iulian Cotoi. Flow patterns in the vicinity of triple line dynamics arising from a local surface tension model. International Journal for Multiscale Computational Engineering, 2007, 5 (5), pp.417-434. 10.1615/IntJMultCompEng.v5.i5.50 . inria-00256573

\section{HAL Id: inria-00256573 \\ https://hal.inria.fr/inria-00256573}

Submitted on 15 Feb 2008

HAL is a multi-disciplinary open access archive for the deposit and dissemination of scientific research documents, whether they are published or not. The documents may come from teaching and research institutions in France or abroad, or from public or private research centers.
L'archive ouverte pluridisciplinaire $\mathbf{H A L}$, est destinée au dépôt et à la diffusion de documents scientifiques de niveau recherche, publiés ou non, émanant des établissements d'enseignement et de recherche français ou étrangers, des laboratoires publics ou privés. 


\title{
Flow Patterns in the Vicinity of Triple Line Dynamics arising from a Local Surface Tension Model
}

\author{
J. Monnier, A.M. Benselama and I. Cotoi
}

November 20, 2006

\begin{abstract}
We model and simulate numerically a droplet impact onto a solid substrate. The triple line dynamics modelling is implicit (as opposed to classical explicit mobility relations), it is based on the Shikhmurzaev equations. These equations include generalized Navier slip type boundary conditions with extra local surface tension gradient terms. Numerical results when spreading are presented. A particular attention is paid to flow patterns near the contact line.
\end{abstract}

\section{Introduction}

One of the main difficulties to simulate present droplet flows is the contact line dynamics modelling. This problem has been widely studied and still remains an open problem. Let us cite macroscopic models with slip boundary conditions, see e.g. [5], [7], [4], mesoscopic models with diffuse interface, see e.g. [9] and also [14], and connection with molecular dynamics, see e.g. [17]. The most frequent contact line model is an explicit mobility relation giving the contact line velocity in function of the wetting angle value at any time (Tanner type laws). Nevertheless, it has been showed, [3], that the dynamic wetting angle cannot be determined inherently by such a mobility relation.

In the present paper, we consider an "implicit" model based on the Shikhmurzaev theory, [16], [3]. This model lead to generalized Navier slip type boundary conditions with local surface tension gradient terms. It does not impose the contact line velocity nor the wetting angle since they are a response of the full model. In Section 2, we present the equations: 2D axi-symmetric Navier-Stokes equations with an ALE formulation. In Section 3, we discretize the equations using a finite element scheme. We introduce a contact line algorithm which imitates caterpillar motion observed in experiments. Curvature is computed using a local Bezier least-square approximation. In Section 4, first we consider a Tanner type law and we test the robustness of our algorithm. Then, we consider both spreading phase and recoiling phase, using the present "implicit" model based on the Shikhmurzaev theory. The extra local terms appearing in this model are set by preliminary results presented in [12] and [13]. We show different flow patterns generated by these terms near the triple point. Finally, we obtain encouraging results for both phases.

\section{Mathematical Model}

\subsection{The equations}

The droplet dynamics is modelled by the2D axi-symmetric unsteady incompressible Navier-Stokes equations. We denote by $\vec{u}=\left(u_{r}, u_{z}\right)^{T}$ the fluid velocity, $p$ its pressure, $\Sigma$ the stress tensor, $D$ the deformation 
tensor and Re the Reynolds number. We denote by $(\vec{\tau}, \vec{n})$ the unit tangential and external normal vectors such that it is direct. We set: $\overrightarrow{\Sigma_{n}}=\Sigma \cdot \vec{n} ; \quad \overrightarrow{\Sigma_{n}}=\Sigma_{n} \vec{n}+\Sigma_{\tau} \vec{\tau}$.

Free surface dynamics and ALE formulation The free surface $\Gamma_{f r}$ is transported by the velocity field $\vec{u}$. It is described by an implicit function: $\phi(t, x(t), y(t), z(t))=0$, and we have:

$$
\frac{d \phi}{d t}=\frac{\partial \phi}{\partial t}+\vec{u} \cdot \nabla \phi=0 \quad \text { in }(0, T) \times \Omega
$$

with given initial conditions.

We present the ALE formulation we use as well as the characteristics method. We refer to [8], [10]. The principle of ALE method is to define an equivalent velocity field $\vec{\gamma}$ (in the sense $\vec{\gamma} \cdot \vec{n}=\vec{u} \cdot \vec{n}$ on $\partial \Omega$ ) such that it preserves the mesh inside the fluid domain $\Omega$. To this end, at any time $t$, we define the deformation field $\vec{\gamma}$ on the free surface $\Gamma_{f r}$, then we extend it all over the domain by solving a linear elasticity system.

Let $t \mapsto C(x, \tau ; t)$ be the characteristics lines associated to the velocity field $\vec{\gamma}$, then one can define the ALE variables: $\vec{u}^{\tau}=\vec{u} \circ C$ and $p^{\tau}=p \circ C$. In the ALE space, fluid particles have a velocity $(\vec{u}-\vec{\gamma})$ at first order in time $(t-\tau)$. And for $t=\tau$ one has: $\frac{\partial \vec{u}}{\partial t}=\frac{\partial \vec{u}^{\tau}}{\partial t}-\vec{\gamma}^{\tau} \nabla \vec{u}^{\tau}$, with $\vec{\gamma}^{\tau}=\vec{\gamma} \circ C$.

If we denote by $t \mapsto X(x, \tau ; t)$ the Lagrangian characteristic lines (i.e. those associated to the velocity field $\vec{u})$, ALE method consists to define a regular field $\vec{\gamma}$ such that: $\Gamma_{f r}^{t}=C\left(\Gamma_{f r}^{0}, t^{0} ; t\right)=X\left(\Gamma_{f r}^{0}, t^{0} ; t\right)$.

The r-momentum equation with ALE formulation is:

$$
\operatorname{Re}\left(\frac{\partial u_{r}^{\tau}}{\partial t}+\left(\overrightarrow{u^{\tau}}-\vec{\gamma}\right) \cdot \nabla u_{r}^{\tau}\right)=-\frac{\partial p^{\tau}}{\partial r}+2\left(\frac{1}{r} \frac{\partial\left(r D_{r r}\left(\overrightarrow{u^{\tau}}\right)\right)}{\partial r}+\frac{\partial\left(D_{r z}\left(\overrightarrow{u^{\tau}}\right)\right)}{\partial z}-\frac{u_{r}^{\tau}}{r^{2}}\right)
$$

The z-momentum equation is similar. The continuity equation is:

$$
\frac{1}{r} \frac{\partial\left(r u_{r}^{\tau}\right)}{\partial r}+\frac{\partial u_{z}^{\tau}}{\partial z}=0
$$

with initial conditions. Boundary conditions on the free surface (liquid-gas) are:

$$
\vec{\Sigma}_{n}=\left(-p_{e x t}+\frac{\kappa^{\tau}}{C a}\right) \vec{n}+h \vec{\tau} \quad \text { in }(0, T) \times \Gamma_{f}
$$

where $C a$ is the Capillary number, $\kappa$ is the mean curvature and $p_{\text {ext }}$ is the external pressure. The extra term $h$ is given (see Shikhmurzaev's theory below). We have classical boundary conditions on the symmetry axis. We decompose the liquid-solid interface into two parts: $\Gamma_{a d}$ and $\Gamma_{s l} \cdot \Gamma_{s l}$ denotes a "small" part of the liquid-solid interface near the triple point. We consider a generalized Navier slip type boundary condition (local slipping):

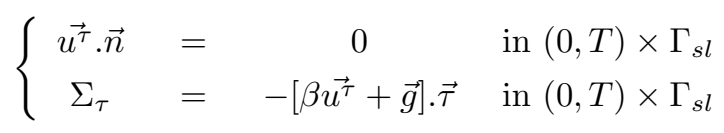

where $\beta$ is a given sliding coefficient. The extra term $\vec{g}$ is given, it models either a surface tension gradient in the Shikhmurzaev's theory or a uncompensated Young stress in the Qian-Wang-Sheng theory, see below.

On $\Gamma_{a d}$, we impose adherence boundary conditions: $\vec{u}=0$.

The solution $\left(\vec{u}^{\tau}, p^{\tau}\right) \circ C$ is an approximation of $(\vec{u}, p)$ at first order in time, and curvatures $\kappa^{\tau}$ approximates $\kappa$ at first order in time too, see e.g. [10].

In all the sequel we simplify notations by dropping the arrows ${ }^{\rightarrow}$ and the superscript ${ }^{\tau}$. 


\subsection{Contact line dynamics modelling}

We consider two different types of model for the contact line dynamics: an explicit model (Tanner type law) and an "implicit" one deriving from Shikhmurzaev theory.

Mobility relation (Tanner type Law). The contact line velocity and the wetting angle are related by:

$$
\left.U_{C L}(t)=k \frac{\left(\theta(t)-\theta^{e q}\right)^{\gamma}}{\left(\theta^{e q}\right)^{\gamma}} \text { for } t \in\right] 0, T[
$$

where $U_{C L}$ is the contact line velocity, $\theta^{e q}$ is the wetting angle at equilibrium (Young's law), $\theta$ is the (dynamic) wetting angle. $k$ and $\gamma$ are parameters usually determined using experimental results.

Local flow modelling and Shikhmurzaev's theory. The "implicit" model does not impose the contact line velocity and the wetting angle but consider them as a response of the model. This model is based on the Shikhmurzaev's theory, [16], [3], which introduces the generalized Navier slip condition (4) and the condition (3). These conditions are local since the extra terms $\vec{g}$ and $h$ vanish except in a vicinity of the triple point. The basic idea of this theory is to consider that the rolling motion observed in experiments, [5], implies that particles of the liquid-gas interface, whom become an element of the solid-liquid, lose their properties in a finite time. Then the surface tension value associated to this particle must change to its new equilibrium value relative to the solid-liquid interface. This process would gives rise to a surface tension gradient in a small vicinity of the advancing contact line (hence a local Marangoni effect). In other respect, the Young equation would remain valid at any time. In this theory,

$$
\vec{g}=-\frac{1}{2 C a} \vec{\nabla} \sigma \text { and } h=\frac{1}{C a} \vec{\nabla} \sigma_{L G} \cdot \vec{\tau}
$$

where $\sigma$ and $\sigma_{L G}$ are the liquid-solid and the liquid-gas surface tension coefficient respectively. In [12] and [13], a mathematical and numerical study presents some qualitative behaviors of $g$ and $h$ arising from Shikhmurzaev's theory.

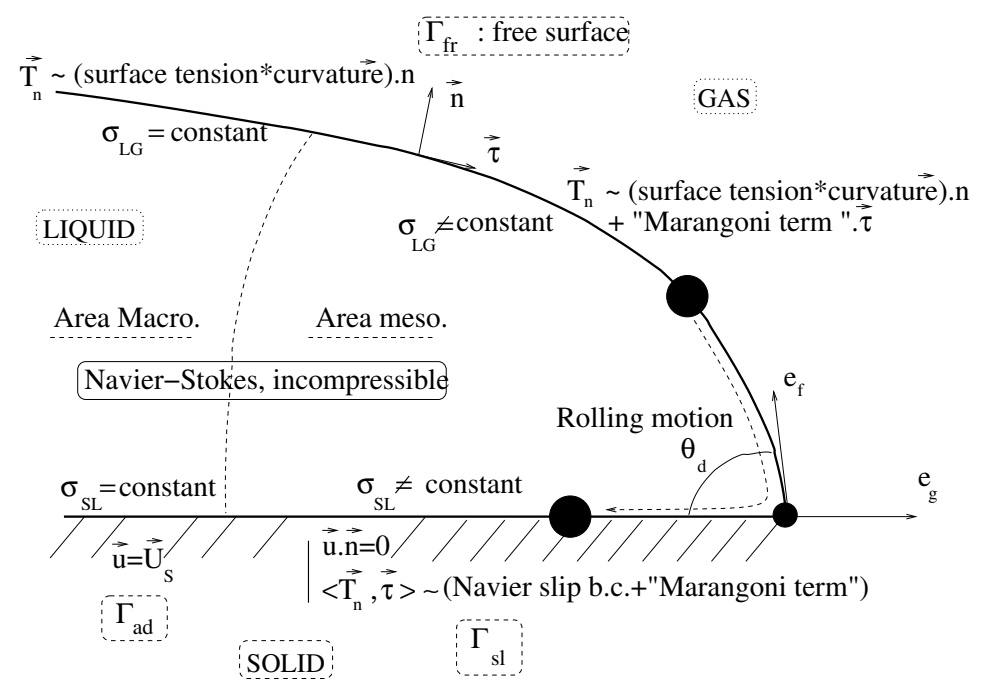

Figure 1: Advancing angle and Shikhmurzaev's model 
The mesoscopic Local Surface Model Briefly, the so-called mesoscopic LSM as it is established in [16] is as follows. The interfaces are described by surface densities $\rho^{s}$ (a variable that describes unambiguously the surface state). These surface densities are solution of surface continuity equations. A linear state equation gives the relation between $\rho^{s}$ and the surface tension coefficients $\sigma$.

We denote: by $\rho_{i}^{s}, i=1,2$, the surface density on $\Gamma_{f r}(\mathrm{i}=1)$ and on $\Gamma_{\text {solid }}(\mathrm{i}=2)$, by $\sigma_{1}=\sigma$ and $\sigma_{2}=\sigma_{S L}$. The surface tension is related to the excess density through the linear state equation:

$$
\sigma_{i}=\gamma\left(\rho_{0}^{s}-\rho_{i}^{s}\right) \quad i=1,2 .
$$

where $\gamma$ and $\rho_{0}^{s}$ are given constants. According to [2], $\gamma \approx 2.10^{6}$ and $\rho_{0}^{s} \approx 10^{-7}$.

We have the surface continuity equation:

$$
\frac{\partial \rho_{i}^{s}}{\partial t}+\operatorname{div}\left(\rho_{i}^{s} \vec{v}_{i}^{s}\right)+\frac{1}{\tau^{*}}\left(\rho_{i}^{s}-\rho_{i}^{e q}\right)=0 \quad i=1,2
$$

where $\tau^{*}$ is the relaxation time relative to the rolling motion, $\tau^{*} \approx 10^{-3}$-see [3]-, $\vec{v}_{i}^{s}$ is a mean velocity inside the layer and $\rho_{i}^{e q}$ is its density at equilibrium, [16]: $\sigma_{i}\left(\rho_{i}^{e q}\right)=\sigma_{i}^{e q}, i=1,2$.

The velocity $\vec{v}_{1}^{s}$ (respectively $\vec{v}_{2}^{s}$ ) is related to $\rho_{1}^{s}$ (respectively $\rho_{2}^{s}$ ) and to the fluid velocity $\vec{u}$. We have the following Darcy laws type, [16]:

$$
\left(1+4 \alpha_{1} \alpha_{2}\right) \vec{\nabla} \sigma_{f r}=4 \alpha_{2}\left(\vec{v}_{1}^{s}-\vec{u}\right) \text { and } \vec{v}_{2}^{s}=\alpha_{1} \vec{\nabla} \sigma_{\text {solid }}+\frac{1}{2} \vec{u}
$$

where $\alpha_{i}, i=1,2$, are given constants characterizing the viscous properties of the interface. According to [2], $\alpha_{1} \approx \frac{h}{\mu}, h$ being the surface layer thick, $h \approx 10^{-10}$.

At the triple line, the surface flux continuity is imposed:

$$
\left(\rho_{1}^{s} \vec{v}_{1}^{s}\right) \vec{e}_{f}=\left(\rho_{2}^{s} \vec{v}_{2}^{s}\right) \vec{e}_{g}
$$

where $\vec{e}_{f}$ and $\vec{e}_{g}$ are unit vectors normal to the contact line and tangential to the gas-liquid and gas-solid interface respectively. Let us notice that: $\cos (\theta)=-\vec{e}_{f} \cdot \vec{e}_{g}$.

Remark. The most important feature of the Shikhmurzaev model is supposed to be the following. It does not impose neither the contact line velocity nor the wetting angle since both are a full response of the model. Nevertheless the LSM is constituted by the two surface equations (8) and the boundary condition (10) at contact line. Then, as it is noticed in ([2]), the condition (10) at contact line is not enough to close the system. To this end the problem misses an extra condition at contact line. This can be very easily showed for some linear second order differential equations with only a flux continuity condition at junction point.

The missing condition cannot be the Young equation otherwise it would imply that the wetting angle must be given, and it would not be a response of the model anymore. The author of [2] proposes an extra condition of the following type:

$$
\left(\rho_{1}^{s} \vec{v}_{1}^{s}\right) \vec{e}_{f}=F\left(\rho_{1}^{s}, \rho_{2}^{s}\right)
$$

where $F(.,$.$) is a bilinear given function related to a chemical potential law.$ 
A connection with Qian-Wang-Sheng theory. From molecular dynamics simulations on immiscible fluids, the authors of [17], [18] show that the relative slipping between the fluid and the solid wall follows a generalized Navier slip boundary condition similar to (4). In this theory, the extra term $\vec{g}$ in (4) would model the contribution of the tangential stress due to non-shear stress that appears because of the anisotropy of the pressure (thus its non-shear origin) across the fluid-fluid interface; normal pressure term is no longer equal to tangential pressure term across this interface, as this usually occurs in the bulk. Hence this term, which is present as far as two(fluid)-phase region is involved, is connected to the fluid-fluid interfacial tension. The extra tangential stress $\vec{g}$, called interfacial uncompensated (or unbalanced) Young stress, is defined as follows: $\int_{\Gamma_{i n t}} g d y=\sigma\left(\cos \theta-\cos \theta^{e q}\right)$, where $\int_{\Gamma_{i n t}} d y$ denotes the integral across the interface $\Gamma_{f r}$.

From a macroscopic point of view, it is obvious that it arises from the deviation of the fluid-fluid interface from its static configuration and is narrowly distributed in the fluid-fluid interface region. Therefore, both theories (Shikhmurzaev's and molecular ones) lead to such a generalized Navier slip boundary condition (equation (4)). The derivation and the interpretation of the extra term $\vec{g}$ is different for each theory but in a mathematical modelling point of view, the boundary condition is similar. Moreover, the authors of [17], [18] point out the fact that, independently of the nearness to the fluid-fluid interface, the generalized Navier slip condition is a more general and suitable condition than the classical non-slip condition, provided slip coefficient $\beta$ and $\vec{g}$ are well adjusted.

\section{Discretization and Algorithms}

\subsection{Finite element scheme}

We discretize the full model in time using the Euler implicit scheme and a first order characteristic method. Let $\left\{t_{0}=0, t_{1}, \ldots, t_{M}=T\right\}$ be a discretization of $] 0, T\left[\right.$ with a constant time step $\Delta t=\frac{T}{M}$. Semi-discrete r-momentum equation is (similar discretisation of z-momentum equation is done):

$$
R e \frac{u_{r}^{m+1}-u_{r}^{m} \circ \chi^{m}}{\Delta t}=-\frac{\partial p^{m+1}}{\partial r}+2\left(\frac{1}{r} \frac{\partial\left(r D_{r r}\left(\vec{u}^{m+1}\right)\right)}{\partial r}+\frac{\partial\left(D_{r z}\left(\vec{u}^{m+1}\right)\right)}{\partial z}-\frac{u_{r}^{m+1}}{r^{2}}\right)
$$

and

$$
R e \frac{u_{z}^{m+1}-u_{z}^{m} \circ \chi^{m}}{\Delta t}=-\frac{\partial p^{m+1}}{\partial z}+2\left(\frac{1}{r} \frac{\partial\left(r D_{z r}\left(\vec{u}^{m+1}\right)\right)}{\partial r}+\frac{\partial\left(D_{z z}\left(\vec{u}^{m+1}\right)\right)}{\partial z}\right)
$$

where the superscript $m$ represent the evaluation of the quantity at $t=t_{m}$ and $\chi^{m}$ denotes the characteristic associated to the velocity field $(\vec{u}-\vec{\gamma})$, defined by:

$$
\left\{\begin{array}{cccc}
\frac{d \chi^{m}}{d t}(t) & = & (\vec{u}-\vec{\gamma})\left(\chi^{m}(t), t\right) & t \in] t^{m}, t^{m+1}[ \\
\chi^{m}\left(t^{m+1}\right) & = & \mathbf{x}
\end{array}\right.
$$

Then, $\chi^{m}(x)$ is approximated by: $\chi^{m}(x) \approx x-\Delta t\left(\vec{u}^{m}-\vec{\gamma}^{m}\right)(x)$.

The semi-discrete z-momentum equation is similar.

We set:

$$
\vec{u}_{a x}=\left(\frac{u_{r}}{r^{2}}, 0\right)^{T}
$$

and we denote by $\operatorname{div}_{r z}(\cdot)=\frac{1}{r} \frac{\partial}{\partial r}(r(\cdot))+\frac{\partial}{\partial z}(\cdot)$. Then we can write (12)-(13) as follows:

$$
\lambda \vec{u}^{m+1}-2 d i v_{r z}\left(\mathbf{D}\left(\vec{u}^{m+1}\right)\right)+\nabla p^{m+1}+2 \vec{u}_{a x}^{m+1}=\lambda\left(\vec{u}^{m} \circ \chi^{m}\right)
$$


with $\lambda=\frac{R e}{d t}$.

Let us recall that $(\vec{u}, p)$ does not denote the Eulerian velocity-pressure but its ALE counterpart.

Equation (1) is semi-discretized using the Euler implicit scheme and the first order characteristic method. This gives:

$$
\phi^{m+1}=\phi^{m} \circ X^{m} \quad \text { in } \Omega, m=1,2 \ldots
$$

where $X^{m}$ denotes the characteristic associated to the velocity field $u$ at time $t_{m}$. It is approximated as follows: $X^{m}(x) \approx x-\Delta t \vec{u}^{m}(x)$.

The space discretization is done using the second order Taylor-Hood finite element. The scheme is implemented using a public $\mathrm{C}++$ finite element library Rheolef, [15].

\subsection{The ALE Free Surface Algorithm}

The free surface problem is solved using an ALE formulation and a characteristics method. The algorithm is the following. At time step $n$, given $\Omega^{n}$ and $\left(\vec{u}^{n}, p^{n}\right)$ in $\Omega^{n}$.

Step 1. Compute the new free surface position $\Gamma_{f}^{n}$ following the Lagrangian characteristic lines (each point of the boundary is translated by $\vec{u}^{n} \Delta t$ ). Then, compute an equivalent deformation field $\vec{\gamma}^{n}$ preserving the mesh by solving a linear elasticity system.

Step 1bis. Compute the new solution $\left(\vec{u}_{\tau}^{n+1}, p_{\tau}^{n+1}\right)$ of Navier-Stokes in $\Omega^{n}$.

Step 2. Update the domain $\Omega^{n+1}=\vec{\gamma}^{n}\left(\Omega^{n}\right)$ (mesh transport by $\vec{\gamma}^{n}$ ).

Step 3. Obtain the new solution $\left(\vec{u}^{n+1}, p^{n+1}\right)$ in $\Omega^{n+1}$ by setting: $\left.\vec{u}^{n+1}\right|_{\Omega^{n+1}}=\left.\vec{u}_{\tau}^{n+1}\right|_{\Omega^{n}}$ and $\left.p^{n+1}\right|_{\Omega^{n+1}}=\left.p_{\tau}^{n+1}\right|_{\Omega^{n}}$.

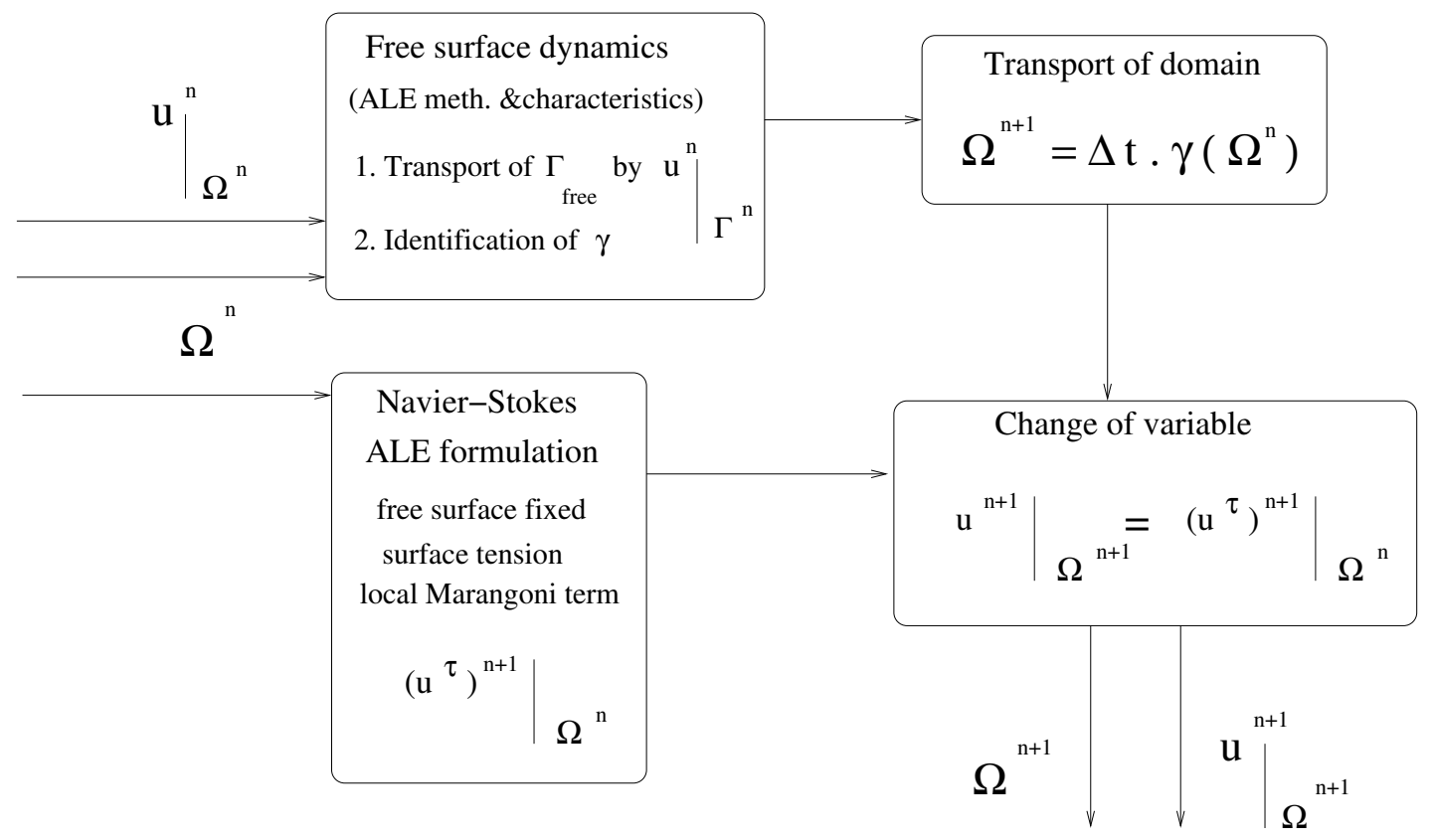

Figure 2: Algorithm of resolution 


\subsection{Spreading and caterpillar motion}

When spreading our algorithm imitates the caterpillar motion observed in experiments, [5]. Fig. 3 represents typical successive time steps. If at time step $n$, the first point (or several points) on the free surface are projected on the solid substrate, all the points on the solid wall but the last ones become no-slip points. The last two points remain slip point.

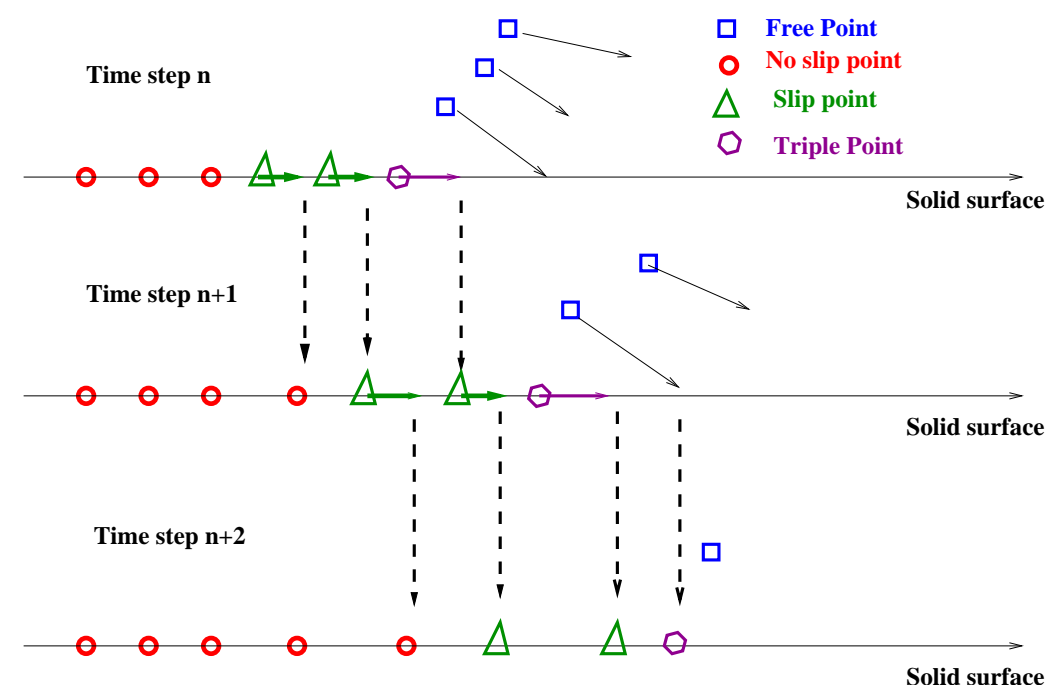

Figure 3: Spreading process. Circles represent no-slip nodes, triangles represent slip nodes $u_{z}=0$, squares represent "free" nodes, diamond is the triple point.

\subsection{Recoiling motion}

When de-wetting (recoiling), the triple point and the slip points are allowed to move horizontally, also vertically if their vertical velocity is positive, see Fig. 4. If the triple point moves away from the solid wall, the first point to the left becomes the triple point, hence becoming a free point. The second point to the left becomes a slip point.

\section{Curvature Computation}

We seek to compute the curvature of the droplet surface. Computing accurately the curvature is a difficult task since its interface is a piecewise linear curve, hence not $C^{2}$ differentiable. In addition, points defining this piecewise linear curve result from the full free surface algorithm, hence may comprises some nonnegligible numerical errors. Then, we seek to estimate the curvature of an underlying smooth surface. Computing a discrete surface curvature is a classical (and difficult) problem. Usually in the CAGD context, surfaces are 3D and triangularized, and the objectives are to smooth the mesh, to simplify it, but not to quantify a local variation of curvature, see e.g. [11].

The present problem is easier since the curve is $2 \mathrm{D}$ only but we are facing the following dilemma: we seek to get rid of numerical errors connected to the points defining the curve while we seek to detect as accurate as possible local variation of curvature, in order to obtain the resulting surface tension forces accurately.

We do not consider a direct computation by a finite difference method since it is very sensitive to data 


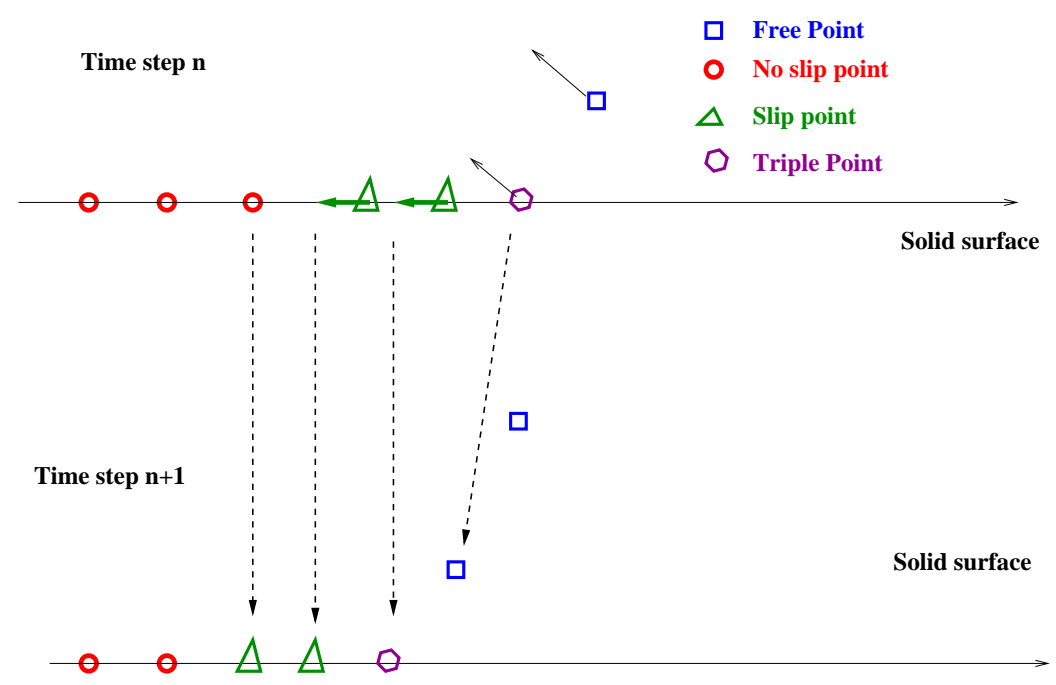

Figure 4: Recoiling process. Circles represent no-slip nodes $\vec{u}=0$, triangles represent slip nodes $u_{z}=0$, squares represent "free" nodes, diamond is the triple point.

error. We do not consider neither a polynomial reconstruction of the underlying smooth surface then evaluate its curvature, since it leads to inaccurate results and unexpected behavior. Following [6], we consider a local least-square approximation then we evaluate the curvature. In the present algorithm, we consider a second order local Bezier approximation. As the numerical tests show (see below), this method filters noise quite reasonably while it allows to detect local and rapid variation of curvature.

\subsection{The Algorithm}

Given $N$ points $X_{i}=\left(r_{i}, z_{i}\right)^{T}, i=1 . . N$, defining the liquid-gas interface, the main idea is to approximate these data using a local least-square approximation by a Bezier's curve.

The Bezier's curve $\mathcal{C}(t)$ is given by:

$$
\mathcal{C}(t)=(r(t), z(t))^{T}=\sum_{j=1}^{M} P_{j} B_{j-1}^{M-1}(t) \quad \text { for } t \in[0,1]
$$

where $P_{j}=\left(\alpha_{j}, \beta_{j}\right)^{T} \in \mathbb{R}^{2}$ are the control points and $\left\{B_{j}^{m}(t)\right\}_{0 \leq j \leq M-1}$ is the classical Bernstein's basis, $B_{j}^{m} \in P_{m}, B_{j}^{m}(t)=C_{j}^{m}(1-t)^{m-j} t^{j}, C_{j}^{m}$ being the binomial coefficients.

We set $M=3$, hence we consider 3 points of control $P_{j}$ and second degree curves.

For an inner point $X_{i}$, see Fig. 5 , we compute the least square approximation of the 5 points $\left\{X_{i-2}, . ., X_{i+2}\right\}$ by a Bezier's curve as follows. We minimize:

$$
J\left(P_{1}, . ., P_{M}\right)=\sum_{l=i-2}^{i+2}\left\|\sum_{j=1}^{M} P_{j} B_{j-1}^{M-1}\left(t_{l}\right)-X_{l}\right\|^{2}
$$

where $\left\{t_{i-2}=0, . ., t_{i+2}=1\right\}$ is an uniform subdivision of $[0,1]$. The unique minimum is computed by solving the corresponding normal equations.

For the extremal point $X_{1}$, we consider a Bezier's curve approximating the points $X_{i}$ for $i=1, \ldots, 4$. For $X_{2}$, we consider a Bezier's curve approximating the points $X_{i}$ for $i=1, . ., 5$. 


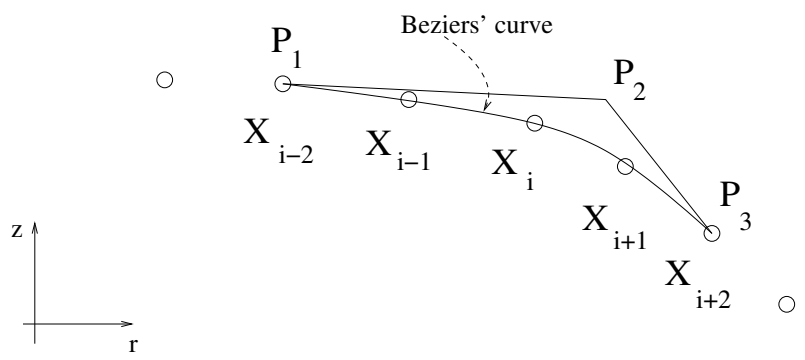

Figure 5: Inner point $X_{i}$. Local least square approximation using Bezier's curve.

For the extremal points $X_{N-1}$ and $X_{N}$, the principle is similar.

Curvature expression. Once a Beziers's curve $\mathcal{C}(t)=(r(t), z(t))^{T}$ is computed for each point $X_{i}$, we evaluate the (plane) curvature as follows:

$$
\kappa_{i}=\kappa\left(t_{i}\right)=\frac{r^{\prime} z^{\prime \prime}-r^{\prime \prime} z^{\prime}}{\left(r^{\prime 2}+z^{\prime 2}\right)^{\frac{3}{2}}}\left(t_{i}\right)
$$

where $\left(r^{\prime}, z^{\prime}\right)$ and $\left(r^{\prime \prime}, z^{\prime \prime}\right)$ are computed using de Casteljau's algorithm, $t_{i}$ being the parameter value corresponding to $X_{i}$.

Sensitivity to random noise. Since the control points defining the (optimal) droplet shape are resulting from the full free surface algorithm, they may be perturbed by some non-negligible numerical errors. Hence, we test the robustness of our algorithm to data perturbation (see below).

If we denote: $N(r, z)=\left(r^{\prime} z^{\prime \prime}-r^{\prime \prime} z^{\prime}\right)$ and $D(r, z)=\left(r^{\prime 2}+z^{\prime 2}\right)^{\frac{3}{2}}$, then: $\kappa(r, z)(t)=\frac{N(r, z)}{D(r, z)}(t)$.

Let $\delta z$ be a perturbation on z-coordinate of data $X_{i}, i=1 . . N$, then we have:

$$
\frac{\partial \kappa}{\partial z}(r, z) \cdot \delta z=\frac{N(r, \delta z)}{D(r, z)}-3 \frac{\kappa(r, z)}{\left(r^{\prime 2}+z^{\prime 2}\right)} z^{\prime} \delta z
$$

This formulae expresses the curvature sensitivity to perturbation on z-coordinate.

Noise introduced in the numerical tests below is a random perturbation (on the z-coordinate of data $\left.X_{i}, i=1 . . N\right)$. It is a normal distribution with zero mean value and unit variance.

\subsection{Numerical tests}

We present some numerical tests using two different methods:

Method a. Standard second order finite difference scheme directly applied to the $\mathrm{N}$ data $X_{i}=\left(r_{i}, z_{i}\right)^{T}, i=$ $1 . . N$,

Method b. Local approximation by a second order Bezier's curve then curvature computation.

In order to compare these two methods, we consider the following three curves (with noise introduced):

Curve 1. a circle, Fig. 6,

Curve 2. an "oscillating-curve", Fig. 7,

Curve 3. a "double circle', Fig. 8. 
As mentioned above, noise is defined as a perturbation on the z-coordinate of data $X_{i}, i=1 . . N$.

The circle is defined by $N$ points as follows: $r(i)=R \cos \left(\eta_{i}\right), z(i)=R \sin \left(\eta_{i}\right)$, with $\eta_{i}=\left(\frac{(i-1) \pi}{2(N-1)}\right)$, $i=1 . . N$.

The so-called "oscillating-curve" is defined by $N$ points as follows:

$r(s)=(R+\epsilon \cos (a . s)) \cos \left(\frac{\pi}{2} s\right), z(s)=(R+\epsilon \cos (a . s)) \sin \left(\frac{\pi}{2} s\right)$ with $s \in[0,1], s$ discretized by $N$ points similarly to $\eta$ and $\epsilon=\frac{R}{10}, a=10$.

The exact curvature of the "oscillating-circle" is straightforwardly obtained.

This curve is interesting since it presents smooth variations of curvature with change of sign.

The so-called "double-circle" is defined by $N$ points as follows:

For $i=1 . . M, r(i)=R \cos \left(\eta_{i}\right), z(i)=R \sin \left(\eta_{i}\right)$; for $i=M+1 . . N, r(i)=a+\frac{R}{2} \cos \left(\eta_{i}\right), a=(1-R) \cos \left(\eta_{M}\right)$ and $z(i)=b+\frac{R}{2} \sin \left(\eta_{i}\right), b=(1-R) \sin \left(\eta_{M}\right)$.

This curve is interesting since it presents a sharp variation of curvature (non-continuous in fact).

We set $R=1, M=8$ and $N=30$.

Without noise (i.e. if considering the exact curves), the two methods allow to retrieve perfectly the curvature values of the three curves.

In figures 6,7 and 8 , we also compare the two methods a. and b. by computing the curvature of curves 1,2 and 3 respectively when some noise is introduced. In such a case, all our computational tests we performed showed that method $b$. gives much better results than method a.

In other respects, we compared methods a. and b. with a "global" method: we interpolate the given points using a parametric cubic spline then we compute the curvature of the interpolating spline. This last method is much less accurate and much more sensitive to noise than methods a. and b.
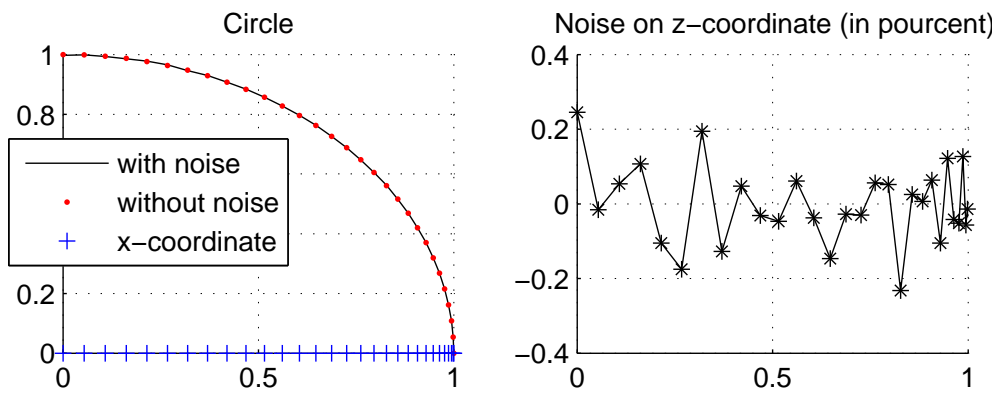

Direct Finite Difference Method

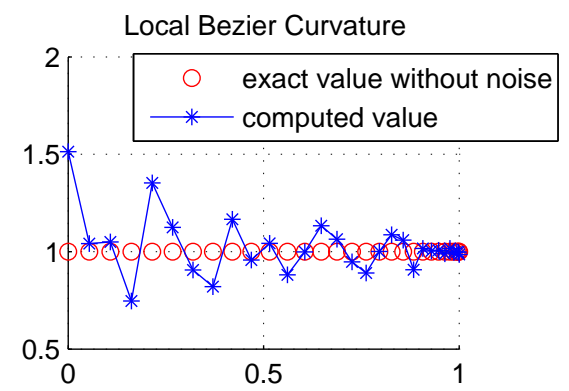

Figure 6: Curve 1. with noise introduced and computed curvature value 

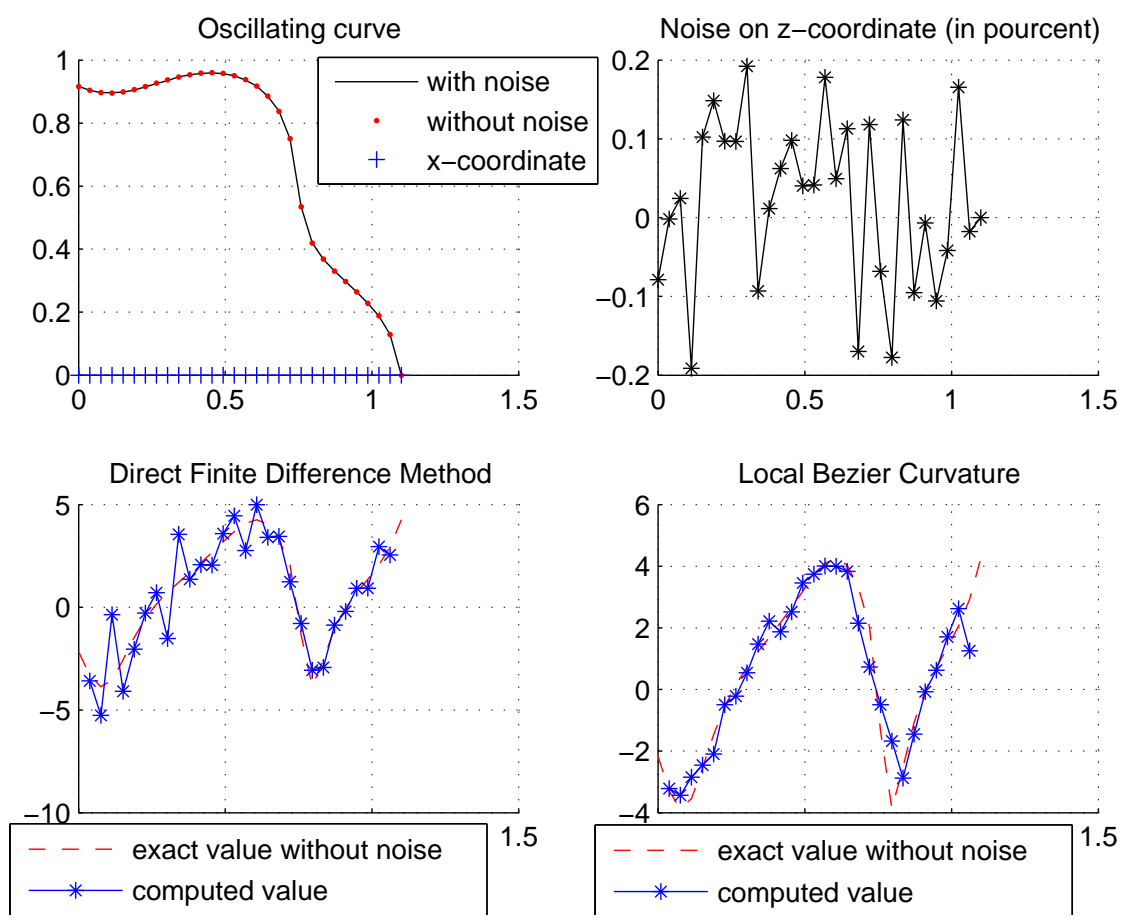

Figure 7: Curve 2. with noise introduced and computed curvature value
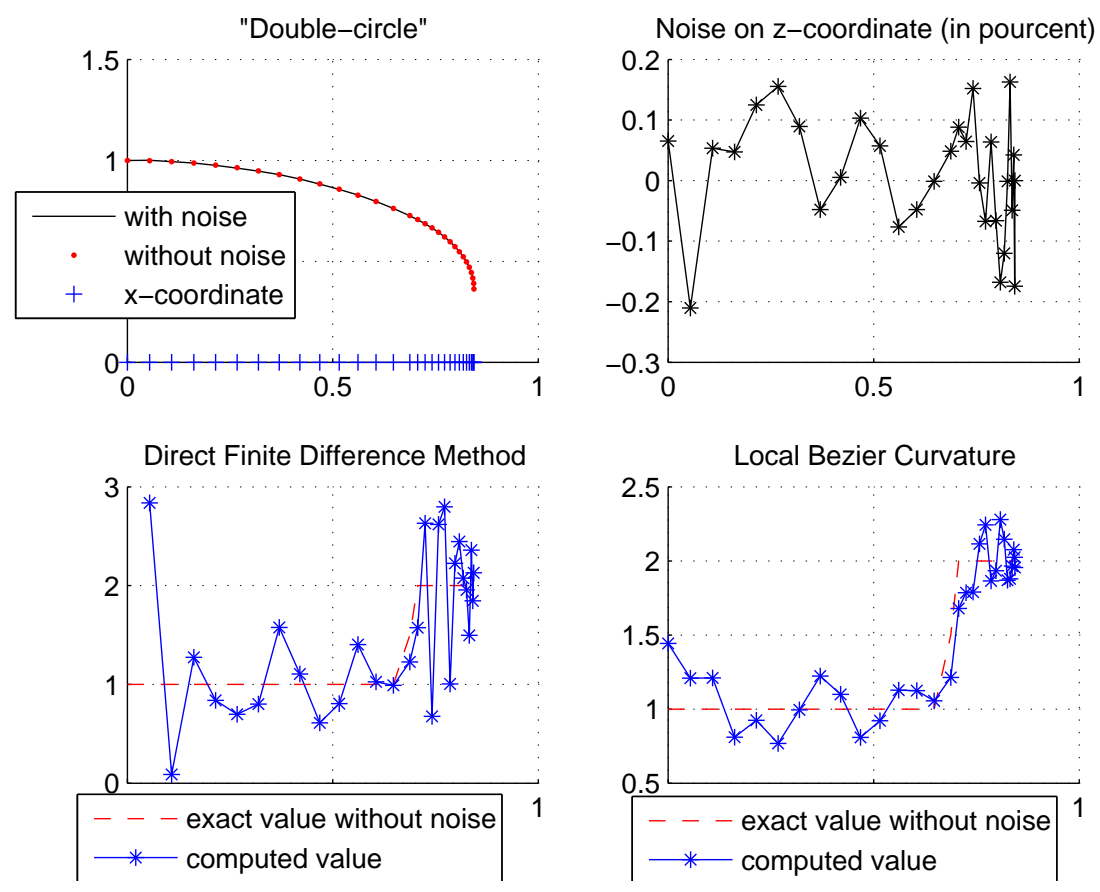

Figure 8: Curve 3. with noise introduced and computed curvature value 


\section{$5 \quad$ Numerical results}

We consider a water droplet. The reference length and velocity are: $L_{r e f}=2.3 \mathrm{~mm}, U_{\text {ref }}=0.98 \mathrm{~m} / \mathrm{s}$. Then, $R e=\frac{\rho U_{r e f} L_{r e f}}{\mu} \approx 46$, Weber number $W e=\frac{\rho U_{r e f}^{2} L_{r e f}}{\sigma} \approx 68$ and $C_{a}=\frac{\sigma}{\mu U_{r e f}} \approx 1.5$.

\subsection{Spreading phase using a Tanner type law}

We start by testing the efficiency of the algorithm. To this end, we consider the Tanner type law as triple line dynamic modelling, and we make fit this law with available experimental data, [1], related to a spreading phase. The triple point position is imposed at each time. We focus on the volume conservation, the height and diameter of the spreading (splat radius) and the deformation of the mesh (number of remeshing necessary), see Fig. 9. With a time step $d t=5 \cdot 10^{-5} s$ and a coarse mesh $(\approx 600$ elements), volume lost after 650 iterations is roughly $3 \%$. The loss occurs mainly at the very beginning of spreading. Mesh transport is efficient hence re-meshing occurs only when a projection occurs (see previous section). From a qualitative point of view, numerical results are similar to experimental data, [1].
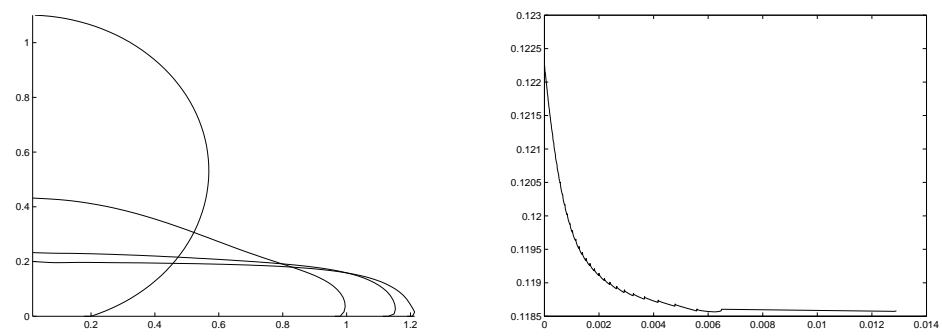

Figure 9: Spreading: droplet profiles and volume in function of time steps

\subsection{Influence of the local parameters $(\beta, g, h)$}

We present some influence of terms specific to the Shikmurzaev's model by considering the following steady-state Stokes system: Find $(\vec{u}, p)$ such that:

$$
\begin{array}{r}
2 \int_{\Omega} D(\mathbf{u}): D(\mathbf{v}) d x-\int_{\Omega} p \operatorname{div}(\mathbf{v}) d x+2 \int_{\Omega} \mathbf{u}_{a} \mathbf{v} d x+\beta \int_{\Gamma_{s l i p}} u_{r} v_{r} d s \\
=\int_{\Gamma_{\text {free }}} \frac{\kappa}{C a} \mathbf{v} \mathbf{n} d s+\int_{\Gamma_{f}} h \mathbf{v} \tau d s-\int_{\Gamma_{s l}} g_{r} v_{r} d s, \quad \forall \mathbf{v} \\
\int_{\Omega} \operatorname{div}(\mathbf{u}) q d x=0, \quad \forall q
\end{array}
$$

We try to find the main effects of parameters $\beta, \vec{g}, h$ from numerical simulations. To do so, we fix one of the three parameters and we simulate the Stokes system (17)-(18) above with different values for the remaining ones. The set of absolute values used for the parameters is $\left\{0,10^{2}, 10^{4}\right\}$ with signs taken as previously discribed. According to Fig. 10 the effect of high $\beta$ values is similar to a no-slip boundary condition on $\Gamma_{s l}$. For the g-term, it is clear that a high $|g|(g<0)$ is equivalent to accelerating the spreading; in order to get the fewer constraint, the tangential velocity have to be of the order of $\frac{-g}{\beta}$. The h-term creates an upwind force which should be responsible for recoiling, see Fig. 11 and 12, for $\beta=0,10^{4}$ and different values of $g$ and $h$. Also, because of continuity, Fig. 10 and 13 suggest the 
presence of a tangential velocity component on the free surface even if coefficient $h=0$, which means that caterpillar-like motion is driven by solid-liquid surface properties as well as by those of the liquid-gas interface. In addition, according to Fig. 12 and $13, \frac{-g}{\beta}$ acts differently on the free interface whether the contact angle is greater or fewer than $\frac{\pi}{2}$.

\subsection{Spreading phase using the Shikhmurzaev model}

We consider the spreading phase using the Shikhmurzaev's model and the algorithm presented previously. As mentioned above, $g$ acts like a control on the position of the triple point. A decrease for $g<0$ translates in a faster spreading. If $g$ is small, this influence is likely not to be seen in the first part of the spreading, when the inertial forces dominate all other forces in presence. By increasing $|g|$ over a threshold, which depends on $\beta$, it accelerates the spreading and modifies the flow nature. For $|g|$ large, large interface distortions occurs and mesh generator fails to re-mesh. In order to observe the influence of the $g$-term, we fix $\beta=1000$, and we perform 1000 iterations with a step size of $d t=5.0 \times 10^{-6}$ for $g \in\{-10,-100\}$, Fig. 14. One can observe that after the inertial phase, the triple point position is farther to the right with an increasing $|g|$ as shown more clearly by Fig. 15 .

Fig. 16 shows a droplet subjected to large distortion (similar to breakup) when $|g|$ is getting larger. Simulation can go no further because of the change of topology it implies is not supported by the ALE method we are using.

We try now to see the influence of the sign of parameter $g$ on the droplet behavior. Since we have seen that parameter $g$ monitor the spreading, it is also supposed to do so with recoiling. In Fig. 17, simulation is started with initially spread droplet. Also here, the ratio $\frac{-g}{\beta}$ significantly influences the behavior of the droplet while parameter $h$ influences more particulary on the droplet curvature than on the triple-point position.

\section{Conclusion}

We have discretized and implemented macroscopic part of the Shikhmurzaev model, [16], [3], using an ALE formulation and a finite element scheme. Also, a connection with Qian-Wang-Sheng's results, [17], has been presented. The present model comprises generalized Navier slip boundary conditions with local surface tension gradient terms in the vicinity of the contact line. The local term values have been chosen upon a previous mathematical and numerical study done in [13]. To test the efficiency of the present algorithm, first we considered a classical mobility relation and we obtained spreading droplet profiles qualitatively comparable to experimental results. Then we showed how the introduction of these new local terms in the vicinity of the contact line allow to model the spreading phase. Concerning the recoiling phase, further investigation to implement properly our algorithm is under progress. Also, a full coupling between the macroscopic part and the microscopic part of the Shikhmurzaev model is under progress.

Acknowledgements. The authors would like to thank A. Soucemarianadin and J.-L. Achard from Laboratory of Geophysical and Industrial Fluid Flows -LEGI- Grenoble, France, for their numerous and fruitful discussions. 


\section{References}

[1] Allaman, S., Desie, G., Vadillo, D., Soucemarianadin, A., Impact and spreading of micro-drops onto solid substrates. Mcaniques \& Industries 4 pp. 443-455 (2003).

[2] Billingham, J., A reappraisal of a model for the motion of a contact line on a smooth solid surface, To appear in Euro. J. Appl. Math. (2006).

[3] Blake, T.D., Bracke, M., Shikhmurzaev, Y.D., Experimental evidence of nonlocal hydrodynamic influence on the dynamic contact angle. Physics of fluids 11 pp. 1995-2007 (1999).

[4] De Gennes, P.G., Wettings: statics and dynamics. Reviews Modern Phys. 57 pp. 827-863 (1985).

[5] Dussan, V.E.B., Davis, S.H., On the motion of a fluid-fluid interface along a solid surface. J. Fluid Mech. 65 pp. 71-95 (1974).

[6] Hamann, B., Curvature approximation for triangulated surfaces, In: G. Farin et al., editor, Geometric modelling, pp. 139-153, Springer-Verlag, (1993).

[7] Hocking, L.M., A moving fluid interface. Part II. The removal of the force singularity by a slip flow. J. Fluid Mech. 79 pp. 209-229 (1977).

[8] Hughes, T.J.R, Liu, W., Zimmermann, T.K., Lagrangian-Eulerian Finite Element Formulation for incompressible Viscous Flows, Comp. Methods Applied Mech. Eng., vol. 29, pp. 329-349 (1981).

[9] Jacqmin D., Contact-line dynamics of a diffuse fluid interface. J. Fluid. Mech., 402 pp. 57-88 (2000).

[10] Maury, B., Pironneau, O., Characteristics ALE method for unsteady free surface flows with surface tension Z. Angew. Math. Mech., 76, pp 613 (1996).

[11] Meyer, M., Desbrun, M., Schrder, P. and Barr., A. H., Discrete differential-geometry operators for triangulated 2-manifolds, In Visualization and Mathematics III, H.-C. Hege and K. Polthier eds., pp. $3557-$, (2003).

[12] Monnier, J., Modélisation Numérique de la Ligne Triple. Internal Report LMC-IMAG, Summer school CEA Grenoble. September (2003).

[13] Monnier, J., Witomski, P., Analysis of a Local Hydrodynamic Model with Marangoni Effect. J. Sc. Comp. 21 pp. 369-403 (2004).

[14] Pomeau, Y., Recent progress in the moving contact line problem: a review. C.R. Mécanique. 330 pp. 207-222 (2002).

[15] Saramito, P., Roquet, N. and Etienne, J., Rheolef, The finite element system, http://wwwlmc.imag.fr/lmc-edp/Pierre.Saramito/rheolef/

[16] Shikhmurzaev, Y.D., The moving contact line problem on a smooth solid surface. Int. J. Multiphase Flow. 19 589-610 (1993).

[17] Qian, T., Wang, X.-P., Sheng, P., Generalized Navier boundary condition for the moving contact line. Comm. Math. Sci. 1 pp. 333-341 (2003).

[18] Qian, T., Wang, X.-P., Sheng, P., Molecular hydrodynamics of the moving contact line in two-phase immiscible flows. Commun. Comput. Phys. 1 pp. 1-52 (2006). 

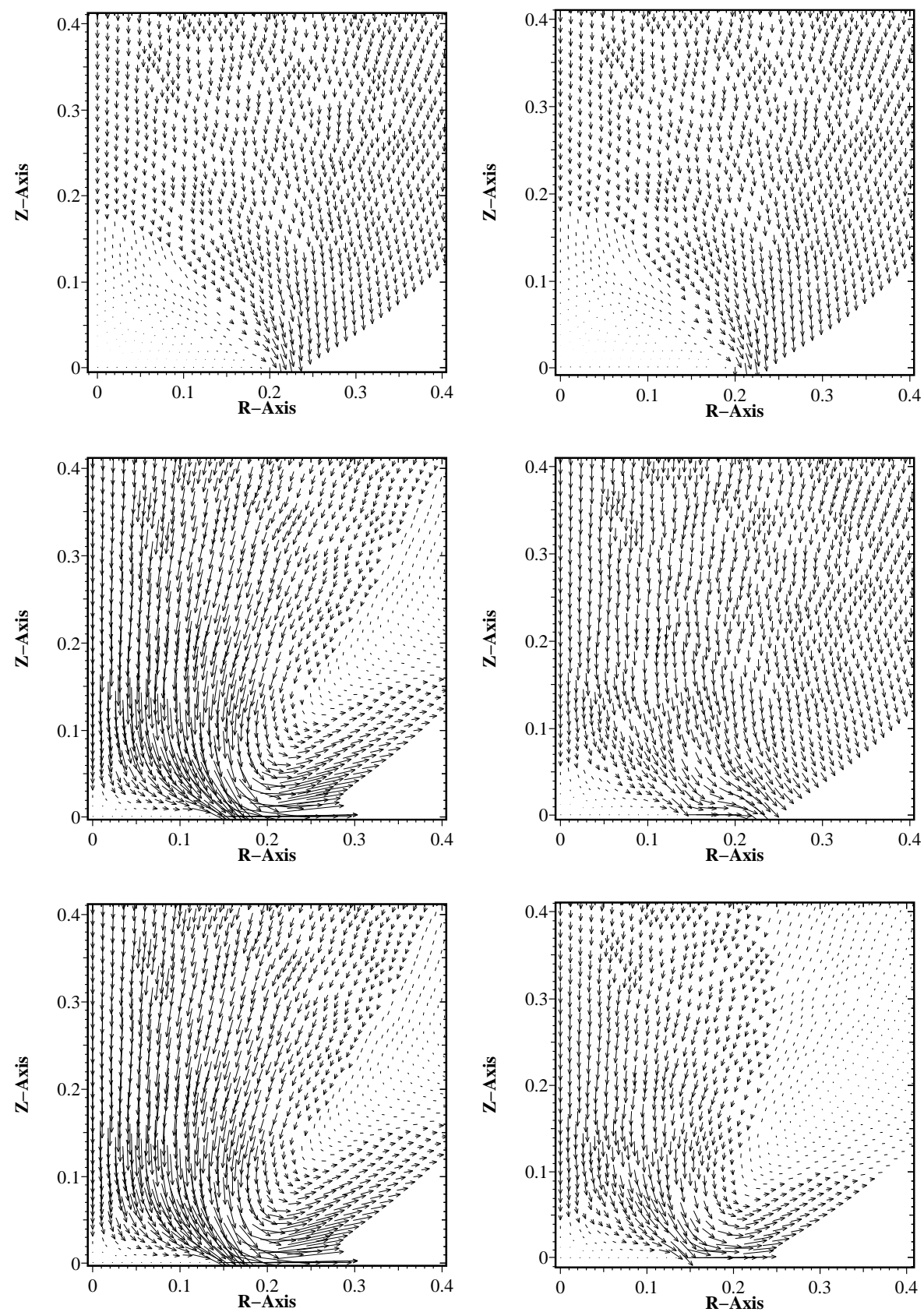

Figure 10: Case 1: influence of $\beta$ and $g, h=0$. Velocity in the vicinity of the triple point for: $(\beta, g)=$ $\left\{(0,0),\left(10^{4}, 0\right)\right\}$ (up), $\left\{\left(0,-10^{2}\right),\left(10^{4},-10^{2}\right)\right\}$ (middle), $\left\{\left(0,-10^{4}\right),\left(10^{4},-10^{4}\right)\right\}$ (down). Scale factors are $3,3,0.05,3,0.0005,0.05$ respectively. 

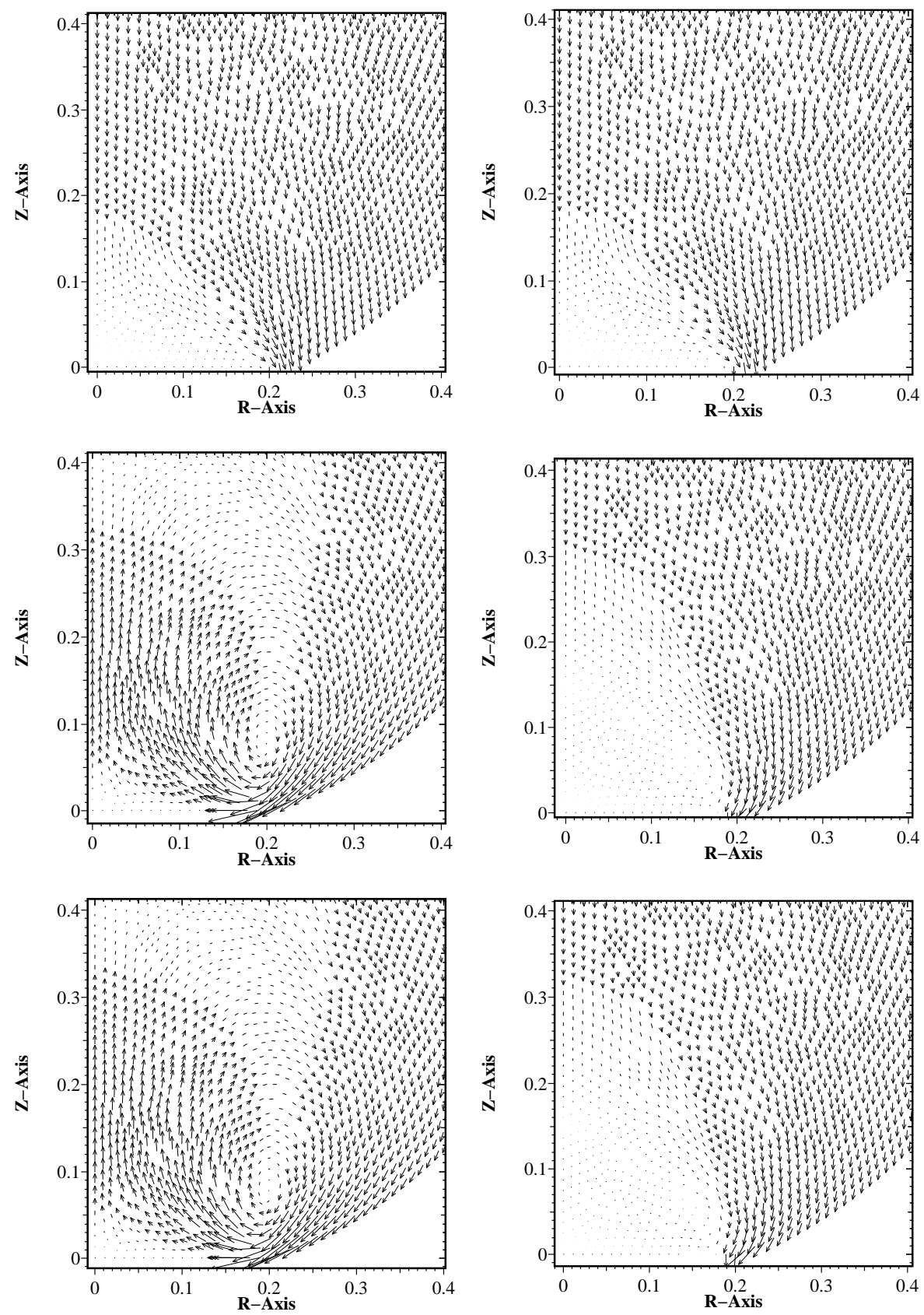

Figure 11: Case 2: influence of $\beta$ and $h, g=0$. Velocity in the vicinity of the triple point for: $(\beta, h)=$ $\left\{(0,0),\left(10^{4}, 0\right)\right\}$ (up), $\left\{\left(0,-10^{2}\right),\left(10^{4},-10^{2}\right)\right\}$ (middle), $\left\{\left(0,-10^{4}\right),\left(10^{4},-10^{4}\right)\right\}$ (down). Scale factors are $3,0.1,0.001$ respectively. 

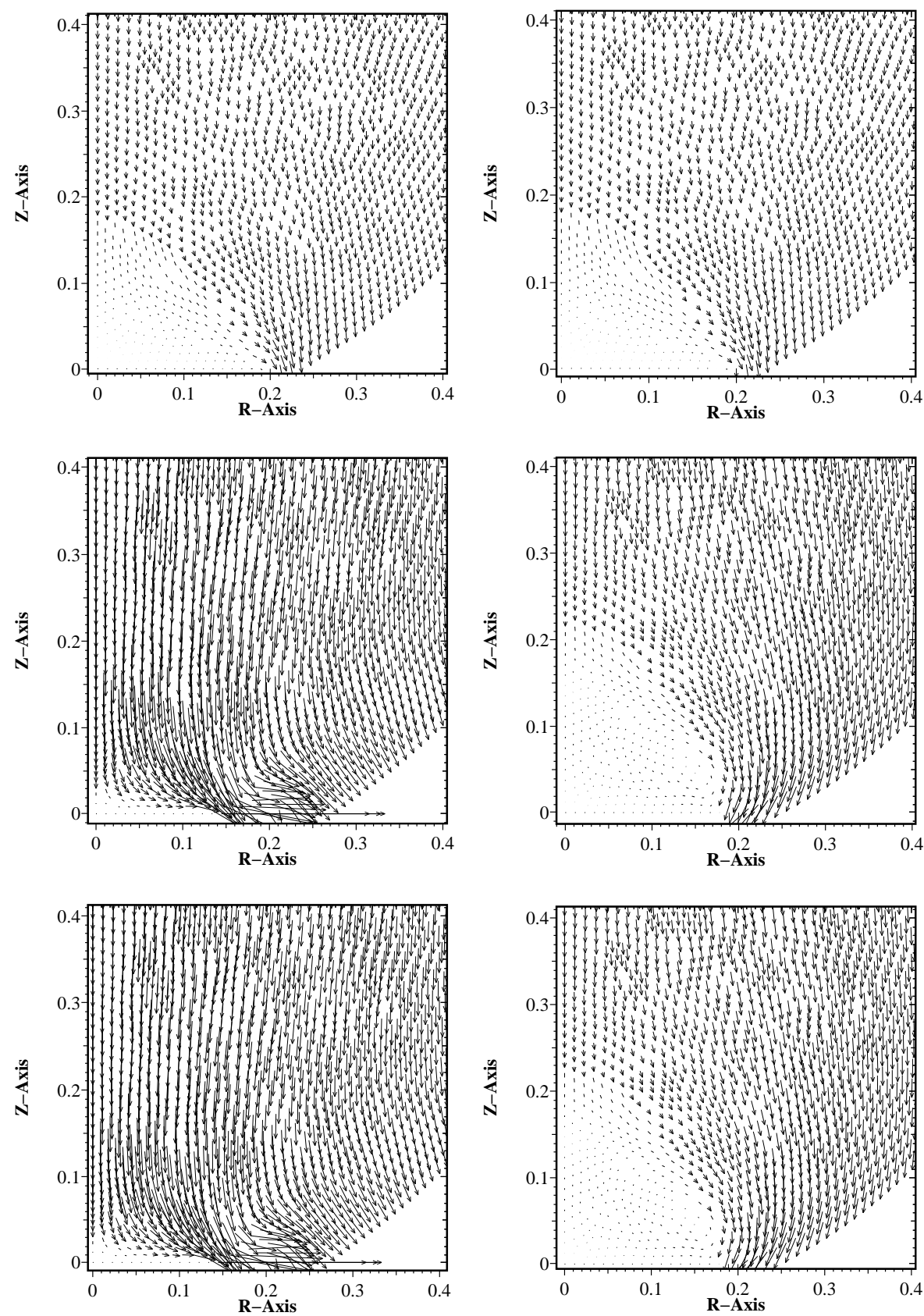

Figure 12: Case 3: influence of $\beta, g$ and $h, h=2 g$. Velocity in the vicinity of the triple point for: $(\beta, g)=\left\{(0,0),\left(10^{4}, 0\right)\right\}$ (up), $\left\{\left(0,-10^{2}\right),\left(10^{4},-10^{2}\right)\right\}$ (middle), $\left\{\left(0,-10^{4}\right),\left(10^{4},-10^{4}\right)\right\}$ (down). Scale factors are $3,0.1,0.001$ respectively. 

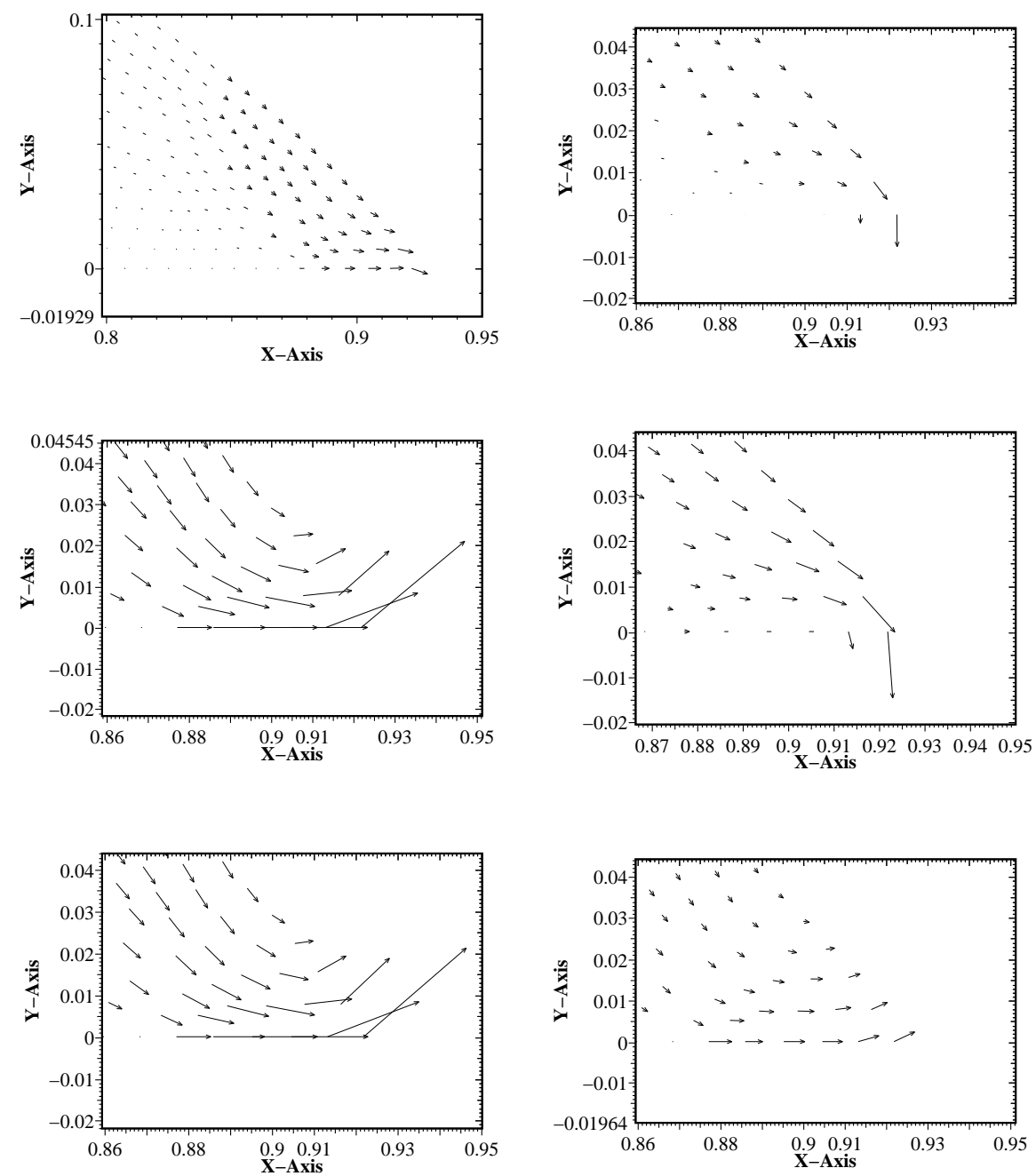

Figure 13: Case 3: influence of $\beta, g$ and $h, h=2 g$. Velocity in the vicinity of the triple point for: $(\beta, g)=\left\{(0,0),\left(10^{4}, 0\right)\right\}$ (up), $\left\{\left(0,-10^{2}\right),\left(10^{4},-10^{2}\right)\right\}$ (middle), $\left\{\left(0,-10^{4}\right),\left(10^{4},-10^{4}\right)\right\}$ (down). Scale factors are $0.05,0.05,0.005,0.1,5 \times 10^{-5}, 0.005$, respectively. $\theta>\frac{\pi}{2}$ 

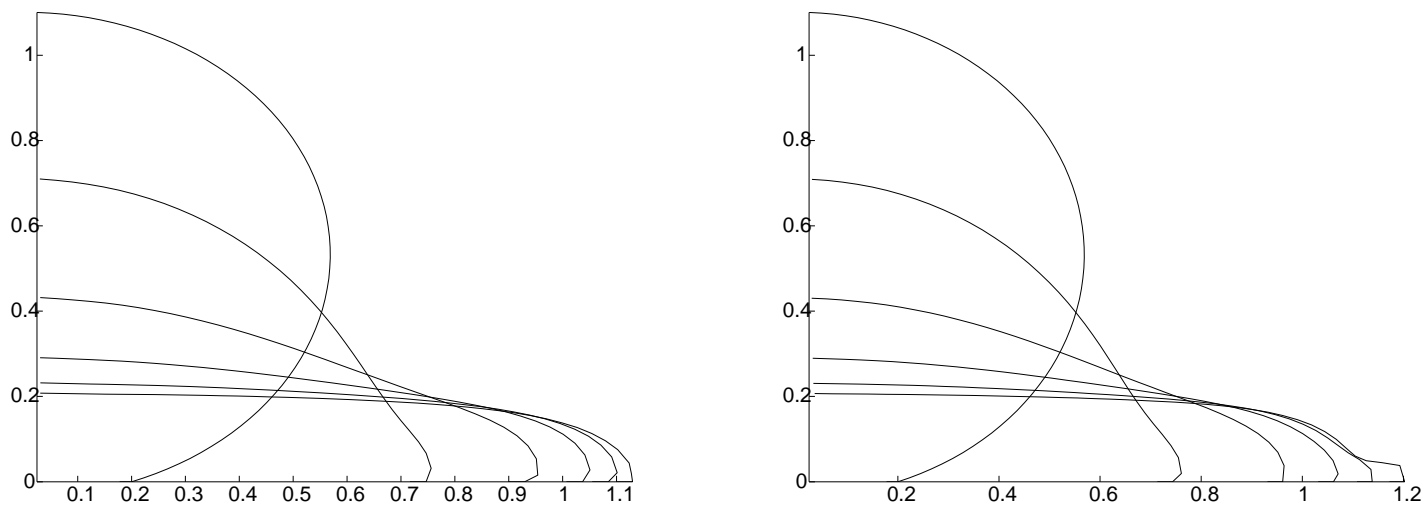

Figure 14: Droplet profiles and triple point position (right) for $(\beta, g)=\left\{\left(10^{3},-10\right),\left(10^{3},-10^{2}\right)\right\}$

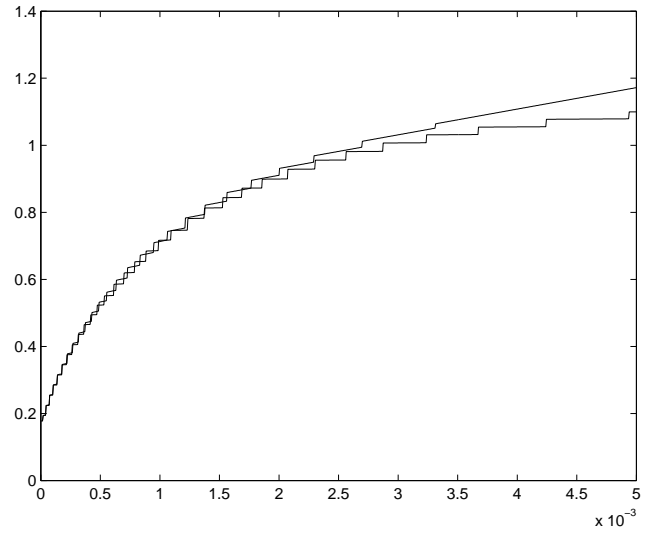

Figure 15: Triple-point porition for $(\beta, g)=\left\{\left(10^{3},-10\right),\left(10^{3},-10^{2}\right)\right\}$ 


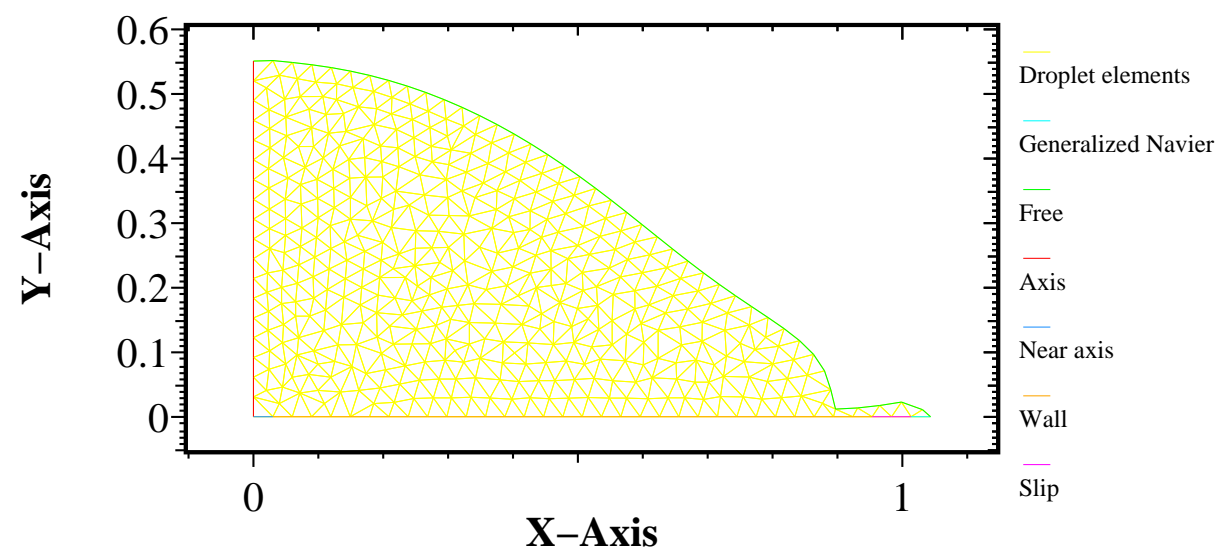

Figure 16: Large interface distortion, similar to a droplet breakup $\left.g-10^{4}\right\}$ 

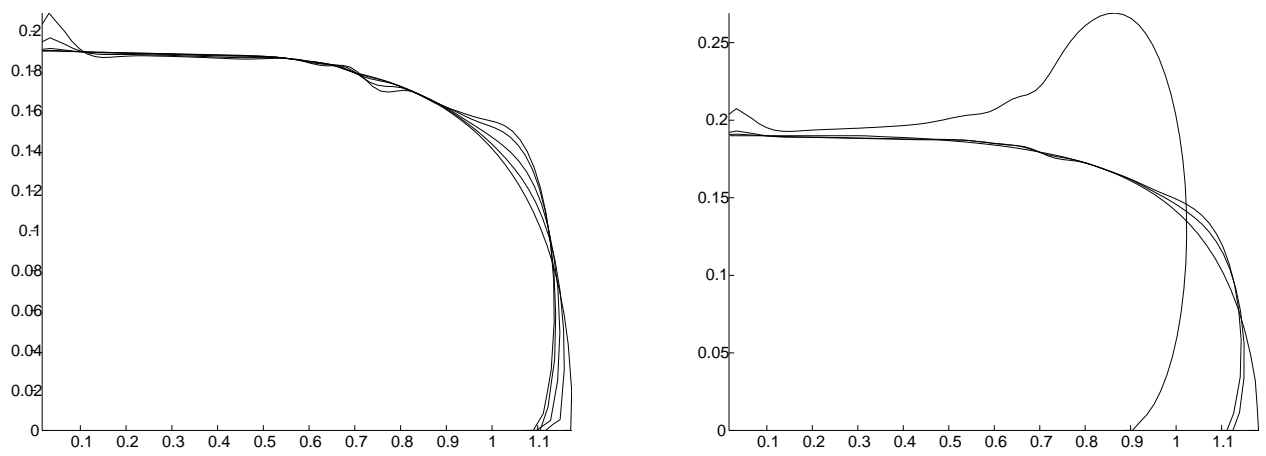

Figure 17: Droplet profiles and triple point position (right) for $(\beta, g, h)=\{(500,100,0),(100,100,-10)\}$ 